Linguistique, littérature, didactique

\title{
Trois notions à l'épreuve de la dimension morale du discours
}

Three Notions Confronted with Moral Dimensions of Discourse

\section{Sophie Moirand}

\section{(2) OpenEdition}

Journals

\section{Édition électronique}

URL : http://journals.openedition.org/pratiques/2303

DOI : $10.4000 /$ pratiques. 2303

ISSN : 2425-2042

\section{Éditeur}

Centre de recherche sur les médiations (CREM)

\section{Édition imprimée}

Date de publication : 31 décembre 2014

\section{Référence électronique}

Sophie Moirand, «Trois notions à l'épreuve de la dimension morale du discours », Pratiques [En ligne], 163-164 | 2014, mis en ligne le 31 décembre 2014, consulté le 19 avril 2019. URL : http:// journals.openedition.org/pratiques/2303 ; DOI : 10.4000/pratiques.2303

Ce document a été généré automatiquement le 19 avril 2019.

(c) Tous droits réservés 


\section{Trois notions à l'épreuve de la dimension morale du discours}

Three Notions Confronted with Moral Dimensions of Discourse

\section{Sophie Moirand}

1 Je tenterai ici de discuter de la dimension morale de la responsabilité énonciative à travers trois catégories qui posent la question de «l'ajustement du discours au monde » (dans un sens quelque peu différent de M.-A. Paveau, 2012: 205-232) et permettent ainsi de débattre des configurations discursives qui en rendent compte :

- il s'agit en premier lieu de la référenciation, en tant que relation du discours au monde, et dans la mesure où intervient la part non linguistique des pratiques langagières où il est produit et interprété, pratiques «relevant des processus d'énonciation» mais aussi " d'activités cognitives non nécessairement verbalisées, pratiques du sujet ou interactions », dans lesquelles « les locuteurs négocient une version provisoire [...] du monde » (Mondada \& Dubois $1995: 84)$;

- or penser la référenciation dans son fonctionnement discursif et historique, c'est-à-dire à travers des catégories qui « ne sont ni évidentes ni données une fois pour toutes » (ibid. : 83), c'est l'aborder en termes de construction des objets de discours, ce qui, au-delà des opérations de désignation/nomination des objets du monde, donc des interactions des locuteurs avec leur environnement, pose, entre autres, la question de la généralisation, "phénomène à la jonction de l'énonciation et de l'argumentation » (Ali Bouacha, 1994: 281);

- cela relève alors pour une part de «la problématique du sujet» (ibid.), voire de la responsabilité énonciative (Moirand, 2006), pour une autre des relations entre les coénonciateurs et leur rapport au monde, donc de l'activité de schématisation qui participe à l'orientation argumentative/pragmatique du discours, telle que l'a conceptualisée J.-B. Grize (1978, 1996, 1998, 2004, 2005 - voir également Miéville, 2010), avec ses notions associées d' éclairage, d'image, de micro-univers et de représentation).

2 Les quelques exemples qui seront ici évoqués au fil du texte ont été saisis pour la plupart « au vol » de propos entendus dans l'environnement, à l'écoute de propos tenus par des 
locuteurs ordinaires et captés à leur insu, ou à l'écoute et à la lecture de l'actualité immédiate mise en forme dans les médias, y compris dans des commentaires enregistrés et/ou postés sur le web.

Ce corpus au vol, complété parfois de propos extraits de corpus constitués antérieurement, me permet d'inscrire cette réflexion sur l'éthique langagière dans un travail récent qui traite plus largement des catégories de l'analyse du discours (Moirand, 2013, 2014 ; Moirand 2015a, 2015b, à paraître).

\section{Référenciation, catégorisation et nomination}

3 Si on pense la référenciation en termes de construction des objets de discours, on remet forcément en cause l'illusion encore très répandue que le langage décrit ou représente le monde tel qu'il est ou tel qu'on le perçoit. On s'interroge alors sur la façon dont le discours catégorise les objets du monde en les "nommant», catégorisations qui «sont plutôt le résultat de réifications pratiques et historiques de processus complexes, comprenant des discussions, des controverses, des désaccords... » (Mondada \& Dubois, 1994 : 283). Or les jugements que l'on peut porter sur la dimension morale du discours, sur certaines désignations ou caractérisations qui surgissent dans l'espace social, et davantage encore dans la mise en discours de l'actualité par le monde politicomédiatique ainsi qu'à travers les tweets et les réseaux sociaux, sont justement induits par cette instabilité, cette légèreté de la parole sociale, en perpétuelle reconstruction.

Dans un texte écrit à deux voix en 2004 (Moirand \& Porquier, 2011), nous avions abordé une première réflexion sur l'éthique langagière à travers l'usage des mots, l'acte de nommer en situation, et en particulier l'emploi du mot otage par des locuteurs divers agacés par des grèves ou autres choses du genre, mot qui perdait alors une partie du sens répertorié par les dictionnaires, alors qu'il le conservait bel et bien lorsqu'on désignait des journalistes pris en otages dans l'intention d'obtenir une rançon, ce que montrent les extraits suivants (archives de la presse en ligne, extraits cités, ibid. : 145-148), usage qu'un journaliste qualifiait d'ailleurs de « traquenard rhétorique » (2):

1. «Otages en Irak. Margaret Hassan lance un appel désespéré aux Britanniques et à Tony Blair »

«Les malades pris en otage »

«Les députés UMP veulent établir un service garanti, pour ne pas prendre les salariés en otage »

2. « Ça me fait penser aux grèves dans les transports, où on entendait cent fois par jour "on est pris en otage". Pas une seule interview sans une grosse femme colérique ou un homme pressé qui ne nous serine sa phrase toute faite, pas un discours politique sans un pro de la communication qui nous l'assène... »

Mais quelque dix ans plus tard, on entend moins les politiques, les médias et les usagers des transports publics dire "nous sommes pris en otage »: les faits survenus depuis (des otages ont été exécutés) ont-ils fini par provoquer un sursaut «moral ", qui empêche de recourir à ce coup de force pragmatique qui consistait à employer le mot à d'autres fins ${ }^{1}$ ?

Cette interrogation sur l'éthique de la nomination rejoignait alors d'autres questions issues d'autres travaux et sur d'autres corpus: certains professionnels du langage (journalistes, enseignants et tout locuteur détenant une parole d'autorité, qui fait que les citoyens ordinaires se reposent sur leurs nominations/désignations, au sens où l'entend L. Kaufmann à travers la notion de déférence), ne devraient-ils pas s'interroger sur l'usage qu'ils font des mots? L'acte de nommer relève-t-il seulement de leur responsabilité 
énonciative ? En tant qu' "énonciateurs dotés d'une parole autorisée ", cela ne relève-t-il pas aussi de leur responsabilité sociale? On sait que les mots sont faits pour circuler, avec toute « l'instabilité lexico-sémantique » (Paveau, 2013 : 31) que cela engendre, mais peuton reprendre la désignation que l'on vient d'entendre ou de lire sans s'interroger sur les conséquences de ses actes de langage? À moins qu'il ne s'agisse de l'acte délibéré d'un discours politicomédiatique préparé ${ }^{2 . .}$

Par la suite, et à la suite de travaux portant sur des polémiques à propos des organismes génétiquement modifiés (OGM), du nucléaire ou du gaz de schiste, ainsi que sur la crise des banlieues de 2005, et plus largement, sur les violences urbaines (Moirand, 2007, 2009, 2014), j'en suis arrivée à la conclusion que, faute de pouvoir intervenir sur l'éthique de la nomination (la recherche du mot propre, c'est-à-dire approprié à ce qu'on sait, ce qu'on voit, ce qu'on sent et ce qu'on veut dire à l'autre), c'est une éthique de l'interprétation des catégorisations opérées par la référenciation qu'il faudrait "penser" dans une perspective de formation citoyenne à la compréhension critique des médias et des discours sociaux, quels qu'ils soient.

7 Or, comme c'est le dire du locuteur qui oriente au moins partiellement l'interprétation, c'est là qu'on en revient à l'énonciation, au choix du locuteur qu'il en soit ou non conscient, pas seulement dans celui des catégories nominales de la référenciation des objets de discours mais également dans celui des cotextes grammaticaux-syntaxiques (à travers des marques de comparaison, de quantification, de coordination, de négation, etc.) qui contribuent à leur éclairage et par suite à l'orientation pragmatique, voire argumentative du discours (au sens de Grize, 2005) ${ }^{3}$ :

3. «Plus de 1000 hectares de maïs transgénique exploités en France »

"OGM : déjà un millier d'hectares en France "

"L'OGM ou la faim?»

[titres]

4. «Le département le plus génétiquement modifié de France... »

«Plus de 20 millions d'hectares d'OGM sont exploités par 8,5 millions d'agriculteurs »

«[...] près de la moitié du maïs et $85 \%$ du soja produits et consommés tous les jours par des millions d'Américains sont des OGM et ne présentent aucun danger»

[éditorial]

8 Dans le même ordre d'idées, ce sont bien les cotextes des énoncés rapportés insérés dans des textes monologaux, ainsi que les cotextes des catégorisations qui stigmatisent par exemple les acteurs de la crise des banlieues, qui contribuent ainsi à l'éclairage qu'on leur donne (Moirand, 2009). Ils illustrent alors parfaitement ce que dit P. Siblot de l'acte de nommer, à savoir que «ce n'est pas seulement se situer à l'égard de l'objet, c'est aussi prendre position à l'égard d'autres dénominations, à travers lesquelles des locuteurs prennent également position » (Siblot, 1997 : 55), et que « la dialogisation interdiscursive est inhérente à la catégorisation, et à l'expression d'un point de vue qu'implique toute actualisation lexicale», comme le montrent ces propos rapportés dans Le Parisien/ Aujourd'hui en France de jeunes « émeutiers » de banlieues :

5. «Rachid et ses compères ont une "tchatche" incroyable.

"Il n'existe pas de brûleurs de voitures heureux"

"Etre casseur n'est pas vraiment une profession. Celui qui met le feu à une voiture est d'abord une victime", renchérit Willy

"A force d'être traité de racaille, on le devient et on le montre [...]. Sarkozy a fabriqué des incendiaires. Il crée des emplois à sa façon", plaisante Rachid ». 
9 Il y a cependant une limite à la « dialogisation interdiscursive » du point de vue d'une éthique langagière, lorsqu'il s'agit de donner des équivalents entre langues et cultures différentes, ou tout au moins des garde-fous dans l'usage que l'on fait d'une mémoire discursive collective non partagée. La question s'est posée à propos de reportages des médias français sur les émeutes urbaines :

- il s'agissait pour la première d'émeutes urbaines en Australie rapportées, photos à l'appui, dans l'hebdomadaire Marianne (24/12/05 : 42) : outre le titre qui met en parallèle la SeineSaint-Denis et Sydney et le sous-titre qui assimile les "émeutes raciales » de Sydney aux "soulèvements de nos banlieues", les correspondants de Marianne à Sydney parlent de "scènes de ratonnades anti-bougnoules" (sans expliquer l'origine et le sens des deux désignations), ce qui relève bien d'une mémoire collective qui date de la guerre d'Algérie pour les Français, mais qui n'a rien de comparable, ni historiquement, ni socialement avec les événements de Sydney - événements et vocabulaire par ailleurs peu «mémorisés " par les générations postérieures aux années 1950-1960 ;

- de même, la presse quotidienne nationale française relatant les manifestations de rue à Athènes en décembre 2008 use des mêmes désignations que celles relatant la crise des banlieues de 2005 et les violences urbaines des manifestations étudiantes de 2006 en France : casseurs, activistes, anarchistes, usant de plus d'un dérivé typiquement français comme smicards pour désigner une catégorie de protestataires proche de celle désignée par la presse grecque comme la génération des 700 euros (traduction du grec), et proposant de même le slogan français datant de Mai 68 (CRS SS) comme équivalent de la traduction du slogan adressé à la police grecque : flics, porcs, assassins. Il ne s'agit plus d'un «ajustement à la réalité » mais bien d'un ajustement à « la culture », et donc à la référenciation qu'on imagine être celle des destinataires... Ce qui est un pari risqué, et cela pose une interrogation éthique aux auteurs de ces "équivalences» dans toute opération de traduction. On ne peut s'empêcher de s'interroger sur la compréhension des éclairages donnés par certaines catégorisations qui, bien entendu, ciblent des destinataires que le locuteur imagine, et non les différentes classes de destinataires que les médias désormais en ligne sont susceptibles d'atteindre. Mais, ce qui me frappe depuis quelques temps, c'est que l'emploi de telles catégorisations n'est pas "expliqué », d'autant qu'elles surgissent souvent dans les titres de presse et les bandeaux qui défilent en bas de l'écran des télévisions, ou dans les formes brèves de l'information sur l'internet et dans les réactions suscitées des publics. Or, ce type de catégorisation, si elle n'est pas suivie d'explication ad hoc, tend à favoriser des généralisations de la part des locuteurs qui les font et surtout par ceux qui les re-prennent, comme on le verra infra (voir supra à propos de voyoucratie).

10 L'importance du cotexte dans l'orientation pragmatique des énoncés est remarquablement montrée par M. Doury et A. Tseronis (2014, à paraître) à propos de la façon dont les politiques sont portés à commenter les résultats lors des débats organisés à la télévision les soirs de résultats électoraux. L'enjeu pour les politiques étant de mettre des mots sur les scores réalisés par les candidats, les auteurs observent, outre le rôle joué par les axiologiques (un score exceptionnel, une sanction sévère, un désaveu incroyable du président sortant), le rôle des «modificateurs quantitatifs» ainsi que celui de configurations discursives particulières: c'est ainsi qu'un score n'est pas «bon » ou «mauvais» en soi; il est construit discursivement comme tel à travers la mise en contexte (l'éclairage donné par le cotexte) des données chiffrées, en pourcentage ou en nombre de votants : 
6. «X qui ne recueille que $25 \%$ des voix » [en fait à ce moment de la soirée : 25,5]

«Vous avez fait moins de $12 \%$ » [en fait $11,7 \%$ à ce moment-là]

«X fait un score proche de $20 \%$ »

«Trois millions : ça fait trois millions de Français qui ont voté pour nous » [en fait

$2 \%]$

«Trois français sur quatre ont voté contre le président sortant » [ils ont voté

pour d'autres]

11 On voit bien que, dans ce genre particulier de débat à la télévision, ce n'est pas une question d'éthique qui intervient mais une question de survie politique (on est ici au soir $\mathrm{du}$ premier tour des présidentielles de 2012). On voit également comment, dans ces opérations de référenciation à des données chiffrées, interviennent les relations des locuteurs au monde et à l'histoire antérieure, et que « l'instabilité des catégories est liée à leurs occurrence dès lors qu'elles sont dans des pratiques » (Mondada \& Dubois, 1995 : 84), ici les pratiques sociales du monde politicomédiatique. C'est donc aux téléspectateurs d'interpréter cette mise en discours des résultats : font-ils appel à une certaine éthique de l'interprétation, qui fait qu'ils ne sont pas dupes de ces présentations, ou ne s'intéressentils qu'aux résultats qui confortent leur propre vote ou leur abstention ? Faut-il, comme le suggère souvent Dominique Wolton, faire confiance aux publics, qui ne seraient pas dupes des professionnels de la parole, médiateurs ou hommes politiques? Ou bien l'éthique de l'interprétation devrait-elle faire partie d'une formation à l'écoute des discours sociaux? C'est cette réflexion que l'on voudrait poursuivre ici à travers la notion de généralisation, qui accompagne souvent l'acte de nommer.

\section{De la catégorisation à la généralisation : une tentation humaine?}

12 Je partirai d'un exemple de généralisation sans «nomination » que j'ai relevée récemment. Attendant un bus qui tardait à venir sous un abribus lors de mon retour à Paris fin juillet, je sentis assez vite une tension monter parmi certains usagers qui comme moi attendaient. Je remarquais alors qu'un homme d'une trentaine d'années sifflait assez fort l'air d'une chanson, un air un peu étrange peut-être pour ne pas dire étranger. Il sifflait assez fort mais, avec le bruit des voitures et motos, ce n'était pas assourdissant. C'est alors qu'une dame dit d'un ton agacé et à haute voix : «I(l)s s'croient tout permis maint'nant ", englobant le siffleur dans un groupe qu'elle pensait inutile de nommer. C'est alors que je m'aperçus que nous avions tous l'air d'européens à peau blanche, sauf le siffleur, sans doute un travailleur pakistanais ou sri-lankais qui, dans ce quartier, sont nombreux à travailler dans les restaurants (à la cuisine généralement). Le siffleur n'est pas monté dans le bus. Interrogeant alors les usagers du bus sur l'interprétation de la dame qui était allée s'asseoir au fond, ils me dirent que le « ils » désignaient forcément les migrants, les immigrants..., voire les réfugiés, les clandestins, voire les roms, sans s'interroger plus avant, certains approuvant par ailleurs la remarque. Le « ils » devenant une indication d'altérité par rapport à " nous », « ils » désignant « les autres » et là plus précisément une catégorie précise, on était en présence d'une généralisation sans nomination, sans catégorisation explicite...

De fait, la généralisation peut s'actualiser à travers la catégorie de la personne : " eux », « ils » « les autres » versus « nous » (voir supra « on est pris en otage »...), et c'est d'ailleurs sur ce type de catégorisation que la Critical discourse analysis s'appuie (entre autres) pour traquer certaines traces de discours idéologique $($ Dijk, 2006) ; elle peut aussi s'actualiser à 
travers des indicateurs de lieu ou de temps: "partout », «toujours» («En Grèce, la nourriture c'est pas terrible, on mange toujours la même chose, je commençais à en avoir assez », disait un Français dans un ferry qui remontait vers Athènes fin juillet), etc. Car si la généralisation peut se définir " comme ce qui permet de dé-construire la singularité d'un événement ou d'une propriété » (Ali Bouacha, 1993a: 50), il s'agit d'examiner «toutes les formes de modulations énonciatives qui pourront affecter l'énoncé sans remettre en cause sa valeur généralisante » soit le rôle des prédéterminants quantifiants, des marques personnelles, des formes aspectuelles du révolu et de l'avenir, des indicateurs spatiotemporels, des opérateurs modaux, etc. (ibid.: 51 sq)... Travaillant actuellement sur les formes brèves des médias, je me suis arrêtée sur deux formes qui me paraissent représentatives de la conception de la généralisation à la frontière de l'énonciation et de l'argumentation, conception que A. Ali Bouacha avait développée dans sa thèse d'État: la question rhétorique, très présente dans les médias (par ex. «Peut-on aimer la guerre? ", titre de une de Libération en août 2014), qu'il a lui-même pris comme exemple de question générique (Ali Bouacha, 1993b), et le fonctionnement actuel des « éléments » pro- et anti- (venus du grec et/ou du latin, dit Le Petit Robert) que je prendrai ici pour exemple.

Regardant les nouvelles sur le site de la chaîne France 24 le samedi 2 août 2014, je trouve l'indication d'une vidéo du jour :

7. «En images : des milliers de pro-Palestiniens défilent à Paris »

Or le fonctionnement des éléments pro et anti dans le discours des médias m'intrigue dans la mesure où il génère une catégorisation en deux camps, qui s'affrontent, au même titre que les questions posées de plus en plus souvent par les médias à leurs lecteurs ou auditeurs internautes (êtes-vous « pour » ou « contre »... - voir Moirand, 2014, 2015b), inscrivant ainsi d'emblée la polémique.

Donc la nomination «pro-palestinien » m'interroge... Est-ce que toutes les personnes qui protestent contre l'offensive à Gaza sont des «pro» Palestiniens? Si l'on prend soin d'écouter la vidéo et/ou d'écouter le reportage qui accompagne la vidéo ou de lire les légendes des photos, on relève une certain nombre de modulations qui relativise ce titre: les caractérisations " mouvement assez hétéroclite », « slogans différents ", " différentes sensibilités ", ainsi que le cadrage donné à la présentation de la vidéo et au texte du reportage :

8. «Selon la police, 11500 personnes ont défilé dans le Centre de Paris, samedi 2

août, pour protester contre l'offensive israélienne à Gaza, lors d'une manifestation autorisée par la Préfecture de police.

Les manifestants appellent à la fin de l'occupation des territoires palestiniens. »

ne semblent pas correspondre à la manière de dire de l'accroche d'ouverture de France 24 , qui englobe l'ensemble des manifestants dans la catégorie " pro-palestiniens ».

Une recherche sur les sites des journaux ainsi que sur la presse imprimée au fil des manifestations suivantes confirment cette impression, si toutefois on prend la peine de lire les textes des brèves ou des articles qui s'y réfèrent: on met cette étiquette sur des partis, des mouvements, des personnalités qui sont contre les bombardements sur Gaza et/ou pour la paix et/ou pour la création de deux États, mais l'appel de titre, les titres, les bandeaux, voire les mots-clés, généralisent en pro et en pour :

9. «Des manifestations pour Gaza sans incidents majeurs » (Le Parisien, 03/08/14).

«À Paris, premier rassemblement pro-gaza après la trève (Le Parisien, 21/08/14). 


\section{israélo-palestinien » ou " pour exiger l'arrêt des opérations militaires dans la bande} de Gaza ».

On voit bien que cette tendance à l'utilisation de l'élément pro- («qui est pour, partisan de » dit Le Petit Robert, qui cite «pro-français, pro-communiste, pro-gouvernemental » et mentionne "par opposition à "anti" »), permet une généralisation qui repose sur «le vague » de la composition : que veut dire être pro-gaza ? Or cette interrogation se renforce au fil des sites consultés : que veut dire une librairie pro-palestinienne dans « une librairie pro-palestinienne saccagée »? Que veut dire un chantage pro-palestinien, attribuée à une chanteuse colombienne à propos de la cérémonie d'ouverture de la Coupe du monde de football : «Coupe du monde 2014 : chantage pro-palestinien de Shakira?»?

Une des dernières consultations que j'ai faite pour ce corpus sur google.fr (le 30/08/2014) permet de vérifier l'étendue des emplois de pro-palestinien et pro-gaza (avec ou sans tirets) dès la première page de références, notamment dans les titres des sites web des journaux français (et à travers les rubriques "Actualités correspondant à pro gaza", "Plus d'actualités pour "pro gaza" ", « Images correspondant à pro gaza »...). Mais les forums où l'on s'interroge sur la définition de cette caractérisation ne rendent pas ces usages moins vagues, et sont assez éloignés de l'article de Wikipedia (à laquelle renvoie le site du dictionnaire collaboratif : dictionnaire.sansagent.com) :

«Cet article est une ébauche concernant la politique :

Pro-palestinien désigne une organisation ou une personne œuvrant à la diffusion et à la culture du peuple palestinien. L'utilisation de ce terme porte une critique intrinsèque selon laquelle le pro-palestinien pondère davantage le bien-être palestinien que israélien. Le langage oppose les pro-israéliens aux propalestiniens. »

D'autant que, à la différence de anti-, qui entre dans la formation de composés attestés en entrée dans le dictionnaire (Le Petit Robert, 2012: 104 sq : anti-américaine, antibourgeois, anticommuniste, anticonstitutionnel, antiféministe, antijeune, antimondialisation, etc.), et glosés par des expressions comme "opposé à l'influence de ", " hostile/hostilité à l'égard de ", "qui s'oppose aux modes de vie et de pensée de", "contraire à», "adversaire du mouvement qui », «mouvement qui s'oppose à », etc., on ne trouve pas de composés en pro- attestés en entrée.

Cependant taper "pro palestinien» sur Google le 30 août 2014 permet d'engranger 495000 résultats en 0,19 secondes et une «Actualité correspondant à propalestinien » (c'est moi qui souligne en gras) :

10. «Le député pro-palestinien George Galloway victime d'une... »

« Actumag Info il y a une heure

Le député britannique George Galloway a été attaqué par un homme visiblement pro-israélien dans la partie Ouest de la ville.»

21 Ce qui incite à consulter Actumag.info sur ce fait divers ainsi raconté :

11. «Le député britannique George Gallloway victime d'une agression sioniste à Londres [titre].

Le député britannique pro-palestinien a été attaqué par un homme visiblement pro-israélien dans la partie ouest de la ville, vendredi 29 août 2014.

George Galloway posait pour une photo avec des fans sur Golborne Road, dans le quartier de Notting Hill, quand un homme a bondi sur lui et a commencé à le frappé. Le député a été immédiatement admis à l'hôpital [...] il serait dans un état relativement mauvais.

Le porte parole du député a déclaré que l'attaque semblait être "en relation avec 
ses propos au sujet d'Israël", en effet l'agresseur hurlait des références sur l'Holocauste.

Le suspect quant à lui a été repéré peu de temps après l'agression et arrêté.

Pour mémoire, George Galloway avait déjà dans le passé été victime d'une agression alors qu'il faisait campagne dans un bus à ciel ouvert à Londres. »

On mesure la distance énonciative qu'il y a entre l'accroche (sur google.fr) et le texte posté sur Actumag.info., et on perçoit le surgissement de l'énonciateur à travers visiblement , sembler, en effet, déjà, etc. Mais si cela n'apporte pas réellement de précision sur le sens de pro- ou d'anti- en contexte (on l'avait déjà remarqué dans d'autres travaux: pro-OGM versus anti-OGM, pro-gaz de schiste versus anti-gaz de schiste, étudiants anti CPE, étudiants antiblocages versus étudiants pro-), cela conduit à s'interroger, outre le rôle de ce type de généralisation catégorielle, sur une troisième catégorie strictement discursive, la schématisation, qui nous renvoie cette fois à l'ordre du discours (voir en 3 ci-dessous), et non plus à l'acte de nommer et/ou de caractériser.

Ainsi des formes de référenciation généralisante divisent le monde en deux camps (et notamment les Français tels que les présentent souvent les médias mais aussi tels qu'ils se présentent eux-mêmes sur certains forums, certains blogs et sur Twitter), à travers des éléments comme pro- ou anti-, des questions ou des énoncés en pour ou contre ou des dénominations rappelant des affrontements historiques (la Fronde des maires s'amplifie [contre les rythmes scolaires], Les transporteurs contre l'écotaxe, Les Français en colère contre leur équipe de foot, Croisade anti-OGM, la résistance s'amplifie [contre le gaz de schiste ou contre le mariage pour tous] $)^{5}$. C'est sans doute efficace du point de vue de la communication, d'autant qu'ils sont particulièrement fréquents dans les éléments paratextuels (légende de photos ou de vidéos, titres, sous titres et intertitres, énoncés détachés, etc.).

La simplification sémantique qui fait qu'on considère, par exemple et pour rester dans le même type d'événement, que les résistants des uns sont les terroristes des autres et vice versa (c'est, parait-il, d'après des étudiants, un exemple fréquent des cours de sémantique discursive...; mais c'est surtout extrêmement présent, implicitement ou non, dans les énoncés brefs, dans les tweets, voire sur les pancartes des manifestants $\left.{ }^{6}\right)$ constitue une autre forme de généralisation qui pose une question éthique au même titre que les oppositions en pro- et anti-, et que la philosophie politique, à défaut des sciences du langage, "ne peut éviter», comme le montre le philosophe et sociologue Gérard Rabinovich, dans son ouvrage Terrorisme/Résistance: d'une confusion lexicale à l'époque des sociétés de masse, ainsi que dans une interview récente accordée à Libération (30 août 2014, propos recueillis par Édouard Launet, p. 30-31), et que je résume ici brièvement, parce que l'étude des généralisations catégorisantes en pro- et anti- me semble relever d'un même type de dé-réalisation du langage (au sens d'activité langagière non située, non historicisée).

Revenant à l'usage et à l'histoire, G. Rabinovich montre comment «terrorisme » et « résistance » sont entrés dans la sémantique politique moderne à la même période, mais à deux moments distincts de la Révolution avant de revenir sur leurs différences historiques et sémantiques et leur évolution. Pour lui «la confusion lexicale » actuelle (« le terrorisme pour les uns est la résistance pour les autres ») « confond deux modalités de guerre, deux mentalités de combat. Il y a dans le terrorisme une "héroïsation de la violence pour elle-même", voire de la mort en fonction d'idéaux, dont il tire gloire. Tandis que dans la résistance, il y a un "consentement" à la violence si elle est inéluctable.» Et comme, pour lui, « le langage comme ordre propre de l'humain s'inscrit 
dans le réel et le transforme ", et que «la pensée n'est pas seulement exprimée par les mots, elle vient à l'existence à travers les mots", il dénonce vigoureusement cette confusion dans les propos rapportés dans Libération: « Ne pas distinguer entre terrorisme et résistance participe d'une anomie lexicale générale, destructrice des aptitudes à penser, conditions de l'autonomie et de la liberté. Une telle anomie est conséquence et vecteur d'une "carence éthique", comme on dit "carence affective" ".

Sans aller forcément dans le sens de ses conclusions, les interrogations qu'il pose relèvent bien d'une éthique du discours, qui s'inscrit dans une réflexion sur «son ajustement au réel », mais également dans le temps de l'histoire et dans la verticalité de la mémoire, et pour nous, linguistes à travers les co-textes des mots, les constructions syntaxiques et les paroles représentées. Dans ses propos recueillis par Libération, G. Rabinovich réagit aux discours circulants, donc aux schématisations qu'il interprète en philosophe, mais cela fait partie de la pensée humaine - $\mathrm{y}$ compris en situation d'interaction-de dénoncer l'amalgame (ce qu'il dit aussi à propos de la paire terroriste/résistant), ou de réfuter le statut d'argument à l'énoncé de l'autre ${ }^{7}$, et cela se manifeste autant dans les interactions en face à face que sur les forums sur l'internet. En ce sens, si s'interroger sur l'éthique langagière revient à interpréter les métadiscours des citoyens ordinaires (Paveau, 2013) autant que les paroles des locuteurs qui font "autorité ", cela me conduit à réfléchir davantage à ce que pourrait être une éthique de l'interprétation et aux conséquences formatives d'une telle position.

\section{Référenciation et schématisation : vers une éthique de l'interprétation?}

25 Je rappellerai à titre d'entrée dans la notion de schématisation, qui me permet de revenir à l'ordre du discours, l'exemple, que j'ai déjà utilisé à d'autres fins, d'un début d'éditorial du Figaro lors des manifestations étudiantes (et enseignantes) du printemps 2006 :

$\mathrm{T} 1:$ : La rue contre les réformes

Cette semaine, les conservateurs sont dans la rue, contre le CPE. Car il ne faut pas se fier aux apparences. Ce sont les porteurs de banderoles qui ne veulent rien changer, défendent le statut quo et s'agrippent à un modèle social remarquable par l'exclusion qu'il engendre aux deux bouts de la vie active - jeunes et seniors. Au point que l'on se demande: pour qui roulent-ils au juste, ces militants de l'immobilisme, sinon pour le maintien de la fracture qui caractérise notre marché du travail entre salariés surprotégés et main d'œuvre surprécarisée ?»

Ce début d'éditorial construit une « représentation » des manifestants à travers « les images » véhiculées par la chaine coréférentielle qui les désignent : conservateurs (par ironie, car ce n'est pas l'image qu'on a des manifestants contre le pouvoir en place), porteurs de banderoles (l'objet emblématique des manifestants, la banderole, contribue à donner un caractère dérisoire à leurs actions - ils sont porteurs de banderoles... et non porteurs des revendications qui y sont inscrites, qu'on ne rappelle pas, et ils sont «la rue », comme le montrent par ailleurs les journaux télévisés du soir, et contre les réformes), donc bien des militants de l'immobilisme, représentation qui concourt au «micro-univers» construit par l'éditorialiste. Cela lui permet d'introduire une assertion imposant au lecteur la représentation que l'auteur généralise à l'ensemble des manifestants sous forme d'une question, que la suite de l'éditorial consiste à justifier. 
27 La schématisation, concept-clé de la logique naturelle proposée par J. B. Grize, permet de revenir à l'ordre du texte et de ne pas s'enfermer dans des catégories qui risquent de le déconstruire (la désignation, l'anaphore, les marques de généralisation, etc.). Elle permet également, comme le suggère J.-M. Adam (Adam, Grize \& Ali Bouacha, 2004: introduction), de concilier la réflexion sur le tout de l'énoncé et celle sur l'interdiscursivité, qu'on voit ici surgir à travers l'image du « modèle social » construite par l'auteur, et censée être celle des manifestants : maintien de la "fracture sociale» (image introduite par le président de la République de l'époque, de droite, lors de sa réélection), opposition entre «les salariés » assimilés aux manifestants et opposés aux « ouvriers » (surprotégé versus surprécarisé), alors que les étudiants manifestent justement contre un contrat précaire qu'on veut leur imposer...

Ainsi lorsqu'un sujet A produit un énoncé, il propose à son interlocuteur B une sorte de reconstruction d'un monde « réel » ou fictif, qui reflète la façon dont lui se représente la situation. La schématisation repose sur la finalité de A mais également sur les représentations que A se fait de B, sur celles qu'il a ou qu'il veut donner de lui-même, et de l'objet dont il parle, le thème $T$. La schématisation, qui est à la fois le processus logicocognitif mis en œuvre par A et le résultat qui est « donné à voir » à B, permet de retrouver les traces de l'activité des sujets. Concrètement elle s'actualise dans le microunivers que le locuteur construit à travers les traces de l'objet de discours, entité discursive repérable à travers des catégories linguistiques comme la thématisation, la recatégorisation, la coréférentialité..., ainsi que les opérations de cadrage (voir Moirand, 2015a).

28 Comment la schématisation permet-elle d'interroger l'éthique langagière ? Quelle rôle à côté de la référenciation et de la généralisation? C'est ce qu'on voudrait discuter ici, à partir d'une définition proposée par H. P. Grize lors du colloque de Dijon, Texte et discours : catégories pour l'analyse (Adam, Grize \& Ali Bouacha, 2004). Définissant la logique naturelle comme l'étude des opérations que la pensée met en œuvre lorsqu'elle se manifeste à travers des discours, il définit alors l'argumentation non pas comme une suite d'arguments mais « comme une organisation raisonnée de contenus de pensée qui vise à modifier de quelques façons les représentations et les jugements de son destinataire » (Grize, 2004 : 23-24).

29 Si l'on conçoit ainsi la schématisation, on verra dans l'information récupérée sur Actumag.info (voir supra, exemple 11) les traces de cette organisation raisonnée (pas forcément consciente) qui intervient dans l'usage qui est fait de pro-X (on a pris à dessein un fait-divers) :

- la représentation de «la victime» est construite à travers plusieurs images, plusieurs catégorisations nominales et des prédicats qui restent assez vagues (tenir des propos au sujet de, déjà victime d'une agression) : il est député, il est britannique, il est pro-palestinien ;

- la représentation de l'agresseur est construite à travers plusieurs images issues pour la plupart de prédicats : un homme visiblement pro-israélien, un homme a bondi et a commencé à frapper, l'agresseur hurlait des références sur l'Holocauste...;

- apparemment le raisonnement qui sous-tend la schématisation ici construite laisse penser que si l'on "hurle des références sur l'Holocauste», on est "visiblement" pro-israélien (on remarquera le raccourci entre l'audition et la vision, la liaison étant assuré par «en effet ») et "déjà » laisse entendre dans la chute du texte "pour mémoire », que la raison de l'agression précédente (il a été déjà agressé) pourrait être de même nature... Mais on voit également comment le titre, qui « annonce » sur Google une " actualité correspondant à pro-palestinien », 
repose sur une autre schématisation, qui efface certaines représentations du député (propalestinien) et transforme des suppositions ou intuitions d'un tiers (semblait être..., dit le porte parole du député selon les propos rapportés), à travers des éclairages introduits par des modificateurs comme visiblement, en effet, déjà, en "agression sioniste " (Le député britannique George Galloway victime d'une agression sioniste). l'occasion de percevoir des images, images évidemment du thème traité, mais aussi de $\mathrm{A}$ et de B, en particulier sous forme de désignation de l'autre et de soi-même. À cela on pourrait ajouter qu'elle donne aussi des images de A même si A ne se « montre » pas, et en particulier dans la construction discursive qui est proposée à B. Elle permet enfin de mettre en parallèle différentes mises en forme de l'actualité, comme un retour sur Google et sur l'agression du député britannique le montre, par exemple le récit proposé par le site Al Huffington Post Algérie, le 30 aout $2014^{8}$ :

12. «George Gallaway, député britannique pro-palestinien agressé brutalement à Londres » [Titre].

31 La schématisation est ici construite à travers des images identiques et des images différentes de celle du texte analysé supra:

- la représentation de l'agresseur: une violente agression commise apparemment par un pro-israélien, un britannique de 39 ans, «il a bondi sur lui et a commencé à le frapper », l'individu a lancé des cris sur l'Holocauste ;

- la représentation de la victime : député britannique, pro-palestinien, âgé de 60 ans, connu pour son engagement pour les Palestiniens ;

- les « raisons » : l'attaque semble liée aux propos du député sur Israël.

13. «Le député a été interrogé la semaine dernière par la police sur les accusations d'incitation à la haine raciale pour avoir déclaré le 2 août dernier sa circonscription de Bradford de [sic] "zone libérée d'Israël" ("Israël-free zone").

Dans son discours prononcée à Leeds, dans un meeting de soutien à Gaza, Galloway a indiqué qu'à Bradford, "nous ne voulons pas de produits israéliens, nous ne voulons pas de services israéliens, nous ne voulons pas que des universitaires israéliens viennent à l'université ou au collège. Nous ne voulons pas que les touristes israéliens viennent à Bradford même si aucun n'a songé à le faire." "

On voit donc ici une schématisation qui ne construit pas seulement une représentation de la victime et de l'agresseur mais tente d'« expliquer », en tout cas de "suggérer », les raisons éventuelles de l'agression. Et c'est à travers les premiers commentaires des internautes qu'on perçoit des interprétations différentes de la schématisation proposée, par exemple à titre d'illustration de réactions de francophones :

14. « Discussion

- il est chez lui, libre, indépendant et décide pour le bienfait de son pays et si Israel est pour lui un « danger » à Bradfort, son devoir de citoyen est tout simplement de dénoncer ce mal...

- Sur tous les grands medias que compte la France aucune information à ce sujet!!!!

quelques sites considérés comme dissidents genre allain jules ou egalite et reconciliation

c'est tout pas de monde figaro et consort

il y a en fait l'info pour les europeens, info pour les africains

- quelle est la réaction de la justice anglaise... ? ? ? on attend

- Quelle qu'en soit la victime, une agression reste un acte plus que réprehensible

- Soutient total. Etonnant au pays de la démocratie ». 
si la notion de schématisation ${ }^{9}$ permet de récupérer l'ordre du texte à partir des choix opérés par l'énonciateur dans les formes de référenciation aux objets du monde et aux relations qui les unissent, elle reste cependant prisonnière d'une conception de l'argumentation qui viserait à «modifier... les représentations et les jugements» des destinataires (Grize, 2004 : 23-24). Or, dans le cas présent, et lorsqu'on analyse certains des propos de locuteurs «ordinaires» ou "autorisés", la définition proposée par M. Doury (à qui j'emprunte par ailleurs la notion de "modificateur») me parait davantage correspondre à ce que je perçois dans les contextes d'apparition des composés avec pro- et anti-, à savoir que « l'argumentation est un mode de construction du discours visant à le rendre plus résistant à la contestation " (Doury, 2014: 76), à condition toutefois que le texte ou l'interaction soit suffisamment longues pour repérer le «micro-univers » que construit la schématisation. Ce qui n'est pas le cas des énoncés pris « au vol » des propos de manifestants (ni des écrits sur les pancartes, ni des slogans repris en boucle), ni des formes brèves de la presse ou des avis des internautes où il s'agit de dire ou d'asserter sans donner les raisons, sans explication ni justification (voir les commentaires reproduits supra - exemple 14).

Il s'agissait dans cette contribution de chercher à répondre à la question que l'on s'était posée à propos du mot « otage » et de quelques autres (Moirand \& Porquier, 2008), que je posai également à la fin d'un travail d'analyse de la presse quotidienne (Moirand, 2007 : 159), et que d'autres se posent à propos des normes en argumentation (Doury, 2014) : porter une attention à l'éthique langagière ne veut pas dire adopter une attitude normative. Il ne s'agit pas de dire comment il faut nommer les choses, si le choix de la nomination est bon ou mauvais, si l'argumentation est bonne ou mauvaise, mais simplement d'apprendre à interpréter les dires de l'autre avec en arrière-plan une réflexion d'ordre philosophique sur l'éthique langagière. Or, dans l'approche des formes du discours, trois catégories m'ont paru intéressantes pour réfléchir à l'orientation pragmatique du discours : la référenciation, la généralisation et la schématisation.

\section{BIBLIOGRAPHIE}

C'est alors une éthique de l'interprétation des discours que l'on voudrait « penser » (voire donner aux autres dans une perspective formative, ou plutôt faire en sorte que les autres se donnent), et « inscrire » dans cette réflexion à entreprendre sur le discours, dans ses rapports tant à l'histoire qu'aux agents et objets du monde et à leurs relations... Finalement, la dimension morale pourrait être une posture de vigilance interprétative à l'égard de toute production de parole, y compris la sienne, en particulier lorsqu'on est en position d'autorité, non pour la censurer, mais pour prendre conscience des forces pragmatiques qui la sous-tendent. Cela constitue de ce fait un objectif à ne pas négliger de l'analyse du discours et des interactions.

ADAM, J.-M., GRIZE, J.-B. \& ALI BOUACHA, A. (éds) (2004) : Textes et discours : catégories pour l'analyse, Dijon, Éd. universitaires de Dijon.

Pratiques, 163-164 | 2014 
ALI BOUACHA, A. (1993a) : "Énonciation, argumentation et discours : le cas de la généralisation », Semen, 8, p. 41-62 (en ligne : http://semen.revues.org/3985).

- (1993b) : «La question générique : statut linguistique et enjeu discursif », in : S. Moirand, A. Ali Bouacha, J.-C. Beacco \& A. Collinot (éds), Parcours linguistiques de discours spécialisés, Berne, P. Lang, p. 279-290.

DIJK, T. van (2006) : « Politique, idéologie et discours », Semen, 21, p. 73-102.

DOURY, M. (2003) : «L'évaluation des arguments dans les discours ordinaires. Le cas de l'accusation d'amalgame ", Langage \& Société, 105, p. 9-36.

- (2014) : La clé argumentative. L'entrée dans les discours par l'argumentation, synthèse pour l'habilitation à diriger des recherches, Université Sobonne Nouvelle - Paris 3.

DOURY, M. \& ASSIMAKIS, T. (à paraître) : «Les fais et les arguments : la mise en discours des scores électoraux », in : J. Goes J., J.-M. Mangiante, F. Olmo F. \& C. Pineira (éds), Actes du colloque Le langage manipulateur : pourquoi et comment argumenter?, Arras, Presses universitaires d'Artois.

GRIZE, J.-B. (1978) : «Schématisation, représentations et images », in : Stratégies discursives, Presses universitaires de Lyon, p. 45-52.

- (1996) : Logique naturelle et communications, Paris, Ophrys.

- (1998) : «Logique naturelle, activité de schématisation et concept de représentation », Cahiers de praxématique, 31, p. 115-125.

- (2004) : « Argumentation et logique naturelle », in : J.-M. Adam, J.-B. Grize \& M. Ali Boucha, Texte et discours : catégories pour l'analyse, Dijon, Éd. universitaires de Dijon, p. 23-27.

- (2005) : « Le point de vue de la logique naturelle », in : M. Doury \& S. Moirand (éds),

L'argumentation aujourd'hui. Positions théoriques en confrontation, Paris, Presses Sorbonne Nouvelle, p. 35-43

HAILON, F. (2013) : «L'événement-argument, support de la circulation idéologique : Villiers-le-Bel (novembre 2007) et la voyoucratie ", in : D. Londei, S. Moirand, S. Reboul-Toure \& L. Reggiani (éds), Dire l'événement. Langage, mémoire, société, Paris, Presses Sorbonne Nouvelle, p. 257-266.

KAUfMANN, L. (2008) : « La société de déférence. Médias, médiations et communications », Réseaux, 148-149, p. 79-116.

KOREN, R. (2006) : « La responsabilité des Uns dans le regard des Autres : l'effacement énonciatif au prisme de la prise de position argumentative ", Semen, 22, p. 93-108.

- (2008) : « Pour une éthique du discours : prise de position et rationalité axiologique », Argumentation et Analyse du discours, 1 (en ligne : http://semen.revues.org/2820 ; consulté le 15 juin 2014).

- (2012) : «Langage et justification implicite de la violence », in : L. Aubry \& B. Turpin (éds), Victor Klemperer. Repenser le langage totalitaire, CNRS Éd., p. 93-105.

- (2014) : Introduction orale à l'atelier « Les nouvelles mises en discours de l'actualité », colloque international Reprises et métamorphoses de l'actualité organisé par le GIS Journalisme à l'Institut d'études politiques de Lyon, 27-28 mars 2014.

MIÉVILLE, D. (éd.) (2010) : La logique naturelle. Enjeux et perspectives, Neuchâtel, Université de Neuchâtel.

MOIRAND, S. (2006) : «Responsabilité et énonciation dans la presse quotidienne : questionnements sur les observables et les catégories d'analyse », Semen, 22, p. 45-60.

- (2007) : Les discours de la presse quotidienne. Observer, analyser, comprendre, Paris, Presses universitaires de France.

- (2009) : « Des façons de nommer "les jeunes" dans la presse quotidienne nationale », 
Adolescence. Revue trimestrielle de psychanalyse, psychopathologie et sciences humaines, t. 27-4, 70, p. 907-919.

- (2013) : «Le dialogisme : de la réception du concept à son appropriation en analyse $\mathrm{du}$ discours ", Cahiers de praxématique, 57, p. 69-100.

- (2014) : «L'hétérogénéité énonciative au fil du texte : la représentation des controverses dans les genres de l'information de la presse quotidienne », Arena Romanistica. Journal of Romance Studies, 14, p. 140-164.

- (2015a, à paraître) : « Le discontinu des catégories linguistiques au risque des genres du discours et du continu de la parole située », Semiotica.

- (2015b, à paraître) : «L'événement saisi par la langue et la communication », Cahiers de praxématique.

MOIRAND, S. \& PORQUIER, R. (2008) : « De l'éthique de la nomination à l'éthique de l'interprétation : autour du mot "otage" et de quelques autres ", in : R. Delamotte-Legrand \& C. Caitucoli, Morales Langagières, Mont-Saint-Aignan, p. 139-153.

MONDADA, L. \& DUBOIS, D. (1995) : « Construction des objets de discours et catégorisation : une approche des processus de référenciation », TRANEL, 23, p. 273-302.

PAVEAU, M.-A. (2006) : Les prédiscours. Sens, mémoire, cognition, Paris, Presses Sorbonne Nouvelle. - (2013) : Langage et Morale. Une éthique des vertus discursives, Limoges, Lambert-Lucas.

RABINOVICH, G. (2014) : Terrorisme/Résistance : d'une confusion lexicale à l'époque des sociétés de masse, Lormont, Éd. Le Bord de l'eau.

SIBLOT, (1997) : « Nomination et production de sens : le praxème », Langages, 127, p. 38-55.

\section{NOTES}

1. Voir également, à propos du mot « otage », Paveau $2006: 113-115$.

2. Sauf à le faire consciemment lorsqu'il s'agit de polémique et de discours politique... lorsque les mots racaille ou voyou furent employés par exemple à des fins de stigmatisation de jeunes manifestants (Moirand, 2009). Fred Hailon montre bien comment l'usage fait par un président de la République du mot voyou et davantage encore du mot voyoucratie, installe de façon délibérée la polémique dans le discours du président. Mais faut-il attendre des travaux de recherche pour se rappeler ses emplois antérieurs (par exemple ceux du début du $\mathrm{XX}^{\mathrm{e}}$ siècle par la droite maurassienne, que rappelle F. Hailon, $2013: 258$, note 7) ? Le retour sur les usages et les locuteurs qui l'ont employé ne peut-il être au moins souligné par les éditorialistes, et pas seulement au hasard de leurs connaissances ou de leurs points de vue, à l'heure où une recherche rapide sur l'internet permet de recenser les emplois antérieurs?

3. Je ne développerai pas ici, l'ayant fait suffisamment ailleurs, le rôle des rappels mémoriels qui surgissent également de l'éclairage donné par certains mots ou certains de leurs cotextes (Moirand, 2007, 2013, par exemple).

4. En ligne: www.france24.com/20140802-paris-France-photos-manifestations-pro-gazapalestininiens-israel-soutien, consulté le 02/08/14.

5. Comme le reconnaissent parfois les médias eux-mêmes, par exemple dans l'article "Affrontement cathodique " dans le supplément «Télévisions» du Monde du 16 mars 2014 à propos de certaines émissions de télévision ou des débats des chaines continues d'information

6. Dans le corpus du 2 août relevé sur France 24 à propos de la manifestation "pro-gaza » autorisée (voir supra), la légende d'une des photos du reportage reprend les paroles de «Fouad, contrôleur de gestion », qui « rejette l'appellation de groupe terroriste utilisé pour désigner le 
Hamas » : « Il s'agit d'un groupe de résistance et c'est pour ça que je suis venu avec un drapeau de la résistance palestinienne. »

7. Sur l'amalgame, on peut consulter les articles de M. Doury (2003) et de R. Koren (2012 et icimême).

8. Accès : www.huffingtonpost.maghreb (consulté le 03/09/14 - apparemment une traduction d'un texte paru sur le site www.theguardian.com.).

9. L'analyse ici proposée est une simplification de la logique naturelle dont on trouvera une version moins édulcorée dans D. Miéville (2013).

\section{RÉSUMÉS}

Cet article discute de la dimension morale de la responsabilité énonciative à travers trois catégories qui posent la question de l'ajustement du discours à la réalité, et qui sont ici mises à l'épreuve de données saisies « au vol » de propos entendus dans l'environnement, ou à la lecture de l'actualité immédiate mise en forme par les médias et la presse en ligne : la référenciation, la généralisation et la schématisation. La réflexion menée à partir de ces trois catégories conduit l'auteure à réfléchir à une éthique de l'interprétation, qui pourrait être une posture de vigilance interprétative à l'égard de toute production de parole, y compris la sienne, une prise de conscience des forces pragmatiques qui la sous-tendent, et qu'on pourrait étendre, dans une perspective de formation citoyenne, à la compréhension critique des discours sociaux.

This paper discusses the moral dimension of enunciative responsibility across three categories that raise the question of the adjusment of discourse to reality when we observe empirical data (newspaper and press on line) : referenciation, generalization and schematization (when speech can be seen as a schematized representation of reality). Starting from these categories, the author reflects on the ethics of interpretation, which could be a posture of vigilance against any speech production, including one's own, and an awareness of pragmatic forces behind it. This ethics could be extended, in a perspective of civil education, to a critical understanding of social discourses.

\section{INDEX}

Mots-clés : éthique de l'interprétation, généralisation, référenciation, responsabilité énonciative, schématisation

Keywords : ethic of interpretation, generalization, enunciative responsibility, schematization

\section{AUTEUR}

\section{SOPHIE MOIRAND}

Université Sorbonne Nouvelle - Paris 3 